\title{
Research Challenges in using Mobile Agents for Data Aggregation in Wireless Sensor Networks with Dynamic Deadlines
}

\author{
Pranesh.V.Kallapur \\ Lecturer \\ Department of Information Technology, \\ National Institute of Technology, Karnataka \\ (NITK), India
}

\author{
Geetha. V \\ Assistant Professor \\ Department of Information Technology, \\ National Institute of Technology, Karnataka \\ (NITK), India
}

\begin{abstract}
Recent research has provided means by which Wireless Sensor Networks (WSN) with dynamic deadlines can make use of mobile agents for data aggregation. Lot of research has already been done with respect to using mobile agents in case of static wireless sensor networks. This paper proposes, along with brief explanation of research issues associated with applying mobile agents, two new techniques to improve the performance of data aggregation using mobile agents. First technique proposed in this paper makes mobile agents 'intelligent' enough to support dynamic wireless sensor networks also. Second technique proposed shows that how multiple mobile agents can also be used, in partitions of the original given wireless sensor which are generated based on some chosen criteria, to reduce overall number of buffer overflows. Paper will be concluded with mentioning of future directions of research.
\end{abstract}

\section{General Terms}

Wireless Sensor Networks, Networks, Sensors, Mobile Agents.

\section{Keywords}

Topology, Mobile Agent, WSN, Scheduling, Dynamic Deadlines, Partitioning.

\section{INTRODUCTION}

Efficient use of energy while maximizing accuracy of data sensing \& aggregation has been very important research area in case of WSNs. This issue becomes of paramount importance in case of hop-by-hop data transfer as sensor nodes near gateway node are likely to go dead quickly than other rendering entire WSN useless. The other issue related to energy consumption is non-uniform variation of phenomenon in different regions of network. Mobile Agents have been proposed as to overcome this problem. Existing Mobility is discussed in $[2,3,5,6]$ whereas explicitly added mobility is proposed in $[4,7,8]$. Major issue is using mobile agents is in defining schedule of their visit to sensor nodes for data aggregation. This problem, called as Mobile Element Scheduling(MES), is analyzed in detail, formulated and proven to NP-Complete in [1].

In the rest of this paper, section 2 gives related work, section 3.1 presents research issues associated with use of mobile agents in WSNs, section 3.2 gives formulation of problem, section 3.3 \& 3.4 present proposed techniques to support dynamic WSNs and reducing buffer over flows using multiple mobile agents. Section 4 gives simulation results for each of these techniques.
Section 5 gives analysis of simulation results. Section 6 outlines research challenges for future work.

\section{RELATED WORK}

Bandwidth criteria based message ferry routing in a sparse network is proposed in [7, 8]. Vehicle Routing Problem (VRP) is proposed in [9] whereas time-window \& periodic variants of same are discussed in $[10,11]$. But, the nature and goal of MES problem is different from those proposed in $[9,10,11]$ even though they seems to be very similar to MES problem. Partition Based Scheduling (PBS) is proposed in [14] and claims to 'prevent' buffer overflows completely. But the MES problem is proven to be inherently NP-Complete in [1]. After giving the proof, reference [1] also propose several heuristic based scheduling algorithms for determining schedule of mobile agent visit to sensor nodes. Techniques for optimizing speed \& energy of mobile agents are proposed in $[15,16]$. Works proposed in [1, $14,15,16]$ make use of single mobile agent irrespective of size of network. Single mobile agent may become infeasible as network size increases leading to increased number for buffer overflows.

In this regard motivation of this paper has been to briefly discuss the research issues in using mobile agents and propose new techniques to solve two of the listed problems to improve the performance of mobile agents.

\subsection{Research Issues in case of Mobile Agent Usage}

Below listed are few major issues with respect to use of mobile agents:

1) Single mobile agents become infeasible as network size increases leading to increased buffer overflows

2) As of now mobile agents support only static networks i.e., they can't learn about new node addition or deletion of existing nodes automatically

3) Most of the existing works make use of single mobile agent in non-dynamic WSNs

4) Mobile agent visiting to sensor nodes doesn't take care about other information like cost of travelling between nodes etc., while aggregating. 
5) Energy efficiency and tour length minimizations of mobile agents are still having scope for improvements

6) Algorithms used for determining visit order to sensor nodes by mobile agents are heuristic based; again there is a lot of scope for research in this regard

7) Minimizing tour length of mobile agent is another direction for research

\subsection{Problem Formulation}

We are given the following:

A wireless sensor network of n nodes: node[1..n]

A matrix $\operatorname{cost}[1 . . \mathrm{n}][1 . . \mathrm{n}]$ that denotes the time taken to go from one node to another

A vector that contains buffer overflow times,

Overflow_time[1..n]. The ith element of this vector determines the time at which the buffer in the ith node in the network will overflow.

$\square$ Overflow Time Threshold Value, overflow threshold

A starting node node 0 .

A heuristic \& dynamic deadline based mobile agent scheduling algorithm to generate sequence of visits to sensor nodes by mobile agent

We make the following assumptions:

The vector overflow time[1..n] consist of integer entries.

$\square$ At time $\mathrm{t}=0$ all the buffers of the sensor nodes start filling up.

The actual data transfer time from the sensor node to the mobile element is negligible.

Scheduling algorithm mentioned in problem formulation above is suitably chosen from [1] as it is, since aim of the paper is not to improve existing algorithm or propose new one. For detailed discussion and illustration of concepts refer to [1]. So, aim of this paper is to:

a) show that mobile agents can support dynamic networks also.

b) show that multiple mobile agents can be applied in reducing overall number of buffer overflows as network size becomes unmanageable by a single mobile agent.

Next two sub sections list the techniques proposed by this paper in the form of algorithms.

\subsection{Algorithm to Support Dynamic Networks by Mobile Agents}

Algorithm being proposed in this section relies on assumption that every sensor node sends a beacon in its antenna range, which is received by all neighbor nodes existing in this range informing about location, overflow time, node id \& battery level etc.

\section{ALGORITHM 1:}

1. Start visiting sensor nodes in the order as generated by chosen scheduling algorithm

2. While visiting each sensor node collect information about changes in its neighborhood in terms of new node addition or existing node going dead
3. If new nodes are added, then incorporate the overflow time of new nodes and find the new visiting sequence as per used scheduling algorithm

4. If existing nodes have gone dead, then find the corresponding deadlines from visit sequence, drop, and again find new sequence of visit to sensor nodes.

For detailed discussion of algorithm working and illustration refer to [17].

\subsection{Algorithm to Make Use of Two Mobile Agents}

The algorithm being proposed in this section tries to partition the network into two parts based on arbitrarily chosen threshold value for overflow time which is given as input to algorithm. Then, a single mobile agent is applied in each partition to visit sensor nodes in the sequence determined the scheduling algorithm chosen.

\section{ALGORITHM 2:}

1. Divide network into two partitions: Partition A containing all sensor nodes with overflow time value below or equal to overflow time threshold value and Partition B containing all other sensor nodes with overflow time values above overflow time threshold

2. For each Partition A \& B generate sequence of visit by using suitable deadline based scheduling algorithm

3. Deploy single mobile agent in each partition and start visiting sensor nodes in the order determined in step 2.

Overflow time threshold chosen above can be determined by experimentation as it will be specific to applications. For detailed discussion refer [18].

\section{SIMULATION \& RESULTS}

Proposed techniques are evaluated through $\mathrm{C} / \mathrm{C}++$ implementation \& simulation. Time of occurrence of any topological change event, time of detection of same event by mobile agent, Number of buffer overflows and Number of Visits to sensor nodes by mobile agent are considered as major parameters of interest for evaluating effectiveness of proposed techniques. As the parameter space is huge, we fixed some of them:

* Grid Size: It is fixed to $100 \times 100$. The speed is taken to be 1 , so the cost values are same as distance between nodes. They were rounded to the nearest integer.

* Simulation time: We simulate for 1800 seconds.

* Location and number of nodes n: The nodes are randomly placed on the grid. We use 10 to 100 nodes in step of 10 nodes each.

* Overflow time values: They are randomly chosen and assigned to sensor nodes.

* Overflow time threshold: Varied over arbitrarily chosen range.

* Lookahead and load balancing factor values (alpha): chosen as 4 and 0.25 respectively for EDF-K lookahead and MWSF algorithms 
Other standard assumptions made in [1] are applicable here also. For the purposes of evaluation, instead of stopping the algorithm when a node missed its deadline, we continue, noting this fact, and updating its deadline. Thus, the metrics used are measured for all sensor nodes in each network topology with size running from 10 to 100 sensor nodes, in the simulation time of 1800 seconds.

\subsection{Results of simulating Algorithm 1}

Table 1. Results of simulating MWSF algorithm with dynamic topology support for various scenarios of topology changes

Single Add:-

SI. No. of nodes added

1

Single Delete:-

SI. No. of nodes deleted

1

Multiple Add:-

SI. No. of nodes added

1

2

Multiple Delete:-

SI. No. of nodes deleted 1 2

Multiple Add and Delete:-

Add:-

SI. No. of nodes added

1

Delete:-

SI. No. of nodes deleted

1

Simultaneous Add:-

SI. No. of nodes added

1

2

Simultaneous Delete:-

SI. No. of nodes deleted

1

2

Simultaneous Add and Delete:-

Add:-

SI. No. of nodes added

1

Delete:-

SI. No. of nodes deleted

1
Overflow time

45

Overflow time

24

Overflow time

45

56

Overflow time

31

2

Overflow time

34

Overflow time

28

Overflow time

45

56

Overflow time

34

5

Overflow time

34

Overflow time

17
Table give below summarizes the different events of new node addition, existing node becoming dead or both occurring at a time and mobile agent learning about these events with respect to simulation time. For detailed description of working of algorithm refer to [17].
Time of addition

244

Time of deletion

484

Time of addition

353

839

Time of deletion

295

565

Time of addition

744

Time of deletion

986

Time of addition

461

461

Time of deletion

1210

1210

Time of addition

897

Time of deletion

897
Time of detection

245

Time of detection

485

Time of detection

354

943

Time of detection

298

566

Time of detection

745

Time of detection

987

Time of detection

462

462

Time of detection

1213

1218

Time of detection

898

Time of detection

906 


\subsection{Results of simulating Algorithm 2}

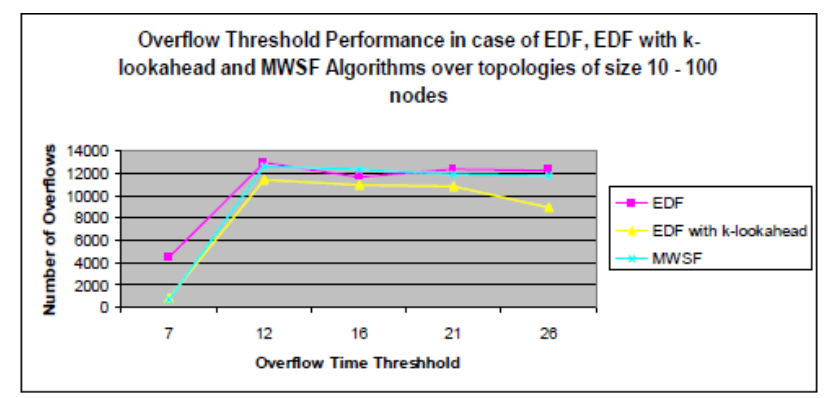

Figure 1 Graph of Average Number of Overflows v/s Overflow Time Threshold for EDF, EDF with klookahead and MWSF algorithms

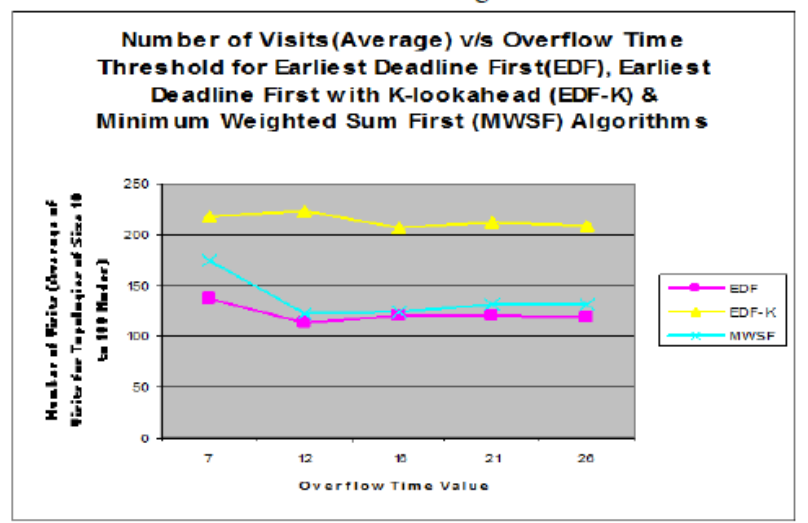

Figure 2 Graph of Average Number of Visits v/s Overflow Time Threshold for EDF, EDF with k-lookahead and MWSF algorithms

For detailed simulation results refer to [18]. Original number of avg. overflows for EDF, EDF-K and MWSF were 13934, 12785 and 13375 respectively. New avg. number of buffer overflows for EDF, EDF-K and MWSF are 10724, 8579, and 9860 respectively, providing 23\%, 32.89 and $26.28 \%$ reduction in number of buffer overflows. Similarly, we can observe the gain achieved by proposed algorithm 2 as original avg. number of visits to sensor nodes for EDF, EDF-K and MWSF were 57, 98, and 64 whereas new avg. number of visits to sensor nodes for EDF, EDF-K and MWSF are 122, 213, and 137 respectively.

\section{ANALYSES OF RESULTS}

The simulation results mentioned in section 4.1 clearly shows that proposed technique makes the mobile agent to learn about topological changes during its current visiting schedule itself than waiting for PE to detect and inform it about these changes. The technique proposed makes the mobile agent to able to enough to learn either single or multiple occurrences of both types of topological changes, namely new node addition or existing node going dead. The performance of scheduling algorithm is unaffected while importing mobile agent this new capability. So, it is very clear that mobile agent can support dynamic networks as well as static networks without much computational and infrastructural expense. Additionally, bandwidth for user data gets increased as hop-by-hop basis topological change information being sent to Processing
Element can be avoided which is surely a great benefit in case of WSNs which happen to be bandwidth constrained most of the times.

From the results given in section 4.2 it can be observed that lower values of overflow time threshold are much helpful in reducing overall number of buffer overflows. This indirectly implies increased number of visits to sensor nodes which can be easily confirmed by looking at graph given in Fig 2. Graphs in section 4.2 also show that increase in threshold value after a particular value, which is specific to application, either won't have any positive effect or can contribute to increase overall number of buffer overflows instead decreasing the same. Also, based on statistical observations mentioned at the end of section 4.2, cost in terms of computation time to partition the network and infrastructural cost associated with additional mobile agent can be ignorable when compared to considerable improvement gained by applying newly proposed technique which, in addition, doesn't affect the performance characteristics of scheduling algorithm much.

\section{CONCLUSION}

Use of mobile agents can be very useful for data aggregation in wireless sensor networks. This paper briefly discussed the research issues while using mobile agents. This paper also presented survey of already proposed two techniques to make mobile agent to support dynamic wireless sensor networks and to reduce overall number of buffer overflows by applying two mobile agents. There is already considerable research going on mentioned research issues for using mobile agents. Scope for further research is vast as mobile agents are promising from point of view of WSNs.

\section{REFERENCES}

[1] Arun A Somasundara, Aditya Ramamoorthy, Mani B Srivastava. Mobile Element Scheduling for efficient Data Collection in Wireless Sensor Networks with Dynamic Deadlines. In Proceedings of the 25th IEEE International Real-Time Systems Symposium (RTSS 2004), 2007

[2] A. Chakrabarti, A. Sabharwal, and B. Aazhang. Using predictable observer mobility for power efficient design of sensor networks. In The second International Workshop on Information Processing in Sensor Networks (IPSN), 2003.

[3] P. Juang, H. Oki, Y. Wang, M. Martonosi, L. Peh, and D. Rubenstein. Energy-efficient computing for wildlife tracking: Design tradeoffs and early experiences with zebranet. In Architectural Support for Programming Languages and Operating Systems (ASPLOS), 2002.

[4] A. Kansal, A. Somasundara, D. Jea, M. Srivastava, and D. Estrin. Intelligent fluid infrastructure for embedded networks. In The Second International Conference on Mobile Systems, Applications, and Services (MobiSys), 2004.

[5] R. C. Shah, S. Roy, S. Jain, and W. Brunette. Data mules: Modeling a three-tier architecture for sparse sensor networks. In IEEE Workshop on Sensor Network Protocols and Applications (SNPA), 2003. 
[6] T. Small and Z. Haas. The shared wireless infostation model a new ad hoc networking paradigm (or where there is a whale, there is a way). In The Fourth ACM International Symposium on Mobile Ad Hoc Networking and Computing (MobiHoc), 2003.

[7] W. Zhao and M. Ammar. Message ferrying: Proactive routing in highly-partitioned wireless ad hoc networks. In The Ninth IEEE Workshop on Future Trends of Distributed Computing Systems (FTDCS), 2003.

[8] W. Zhao, M. Ammar, and E. Zegura. A message ferrying approach for data delivery in sparse mobile ad hoc networks. In The fifth ACM International Symposium on Mobile Ad Hoc Networking and Computing (MobiHoc), 2004.

[9] P. Toth and D. Vigo, editors, "The Vehicle Routing Problem", Society for Industrial \& Applied Mathematics (SIAM), 2001.

[10] M. Solomon, "Algorithms for the vehicle routing and scheduling problem with time window constraints", Operations Research, 35(2), Mar-Apr 1985.

[11] P. Toth and D. Vigo editors, " The Vehicle Routing Problem", Society for Industrial \& Applied Mathematics (SIAM), 2001.

[12] www.jamesreserve.edu (james san jacinto mountains reserve
[13] N. Bansal, A. Blum, S. Chawla, and A. Meyerson, " Approximation algorithms for deadline-tsp and vehicle routing with time-windows", In 36th ACM Symposium on Theory of Computing (STOC), 2004.

[14] Yaoyao Gu, Doruk Bozdagॅ, Eylem Ekici, Fu“sun O“ zgu"ner, Chang-Gun Lee, "Partitioning Based Mobile Element Scheduling in Wireless Sensor Networks", IEEE International Conference on Mobile Computing, 2005.

[15] Zheng wei, Liu sanyang, Kou xiaoli, and Qi xiaogang, "Mobile agent routing algorithm in dynamic sensor network", IEEE International Conference on Communications and Mobile Computing, 2010.

[16] Ryo Sugihar, and Rajesh K. Gupta, "Optimal Speed Control of Mobile Node for Data Collection in Sensor Networks", IEEE Transactions On Mobile Computing, Vol. 9, No. 1, January 2010.

[17] Pranesh V Kallapur, and Niranjan N Chiplunkar, "Topology Aware Mobile Agent for Efficient Data Collection in Wireless Sensor Networks with Dynamic Deadlines", IEEE Conference on Advances in Computer Engineering (ACE), June 2010.

[18] Pranesh V Kallapur, and Niranjan N Chiplunkar, "Multiple Mobile Agents for Data Collection in Overflow Time Based Partitions of Wireless Sensor Networks with Dynamic Deadlines", R10 Asia-Pacific regional IEEE Conference on Advances in Communication Technology (ICACT), February 2011 